\title{
Compressible Pipe Flow with Friction and Gravity
}

\author{
Etsuo Morishita ${ }^{*}$ \\ Meisei University, Department of Mechanical Engineering, 2-1-1 Hodokubo, Hino, Tokyo, 191-8506 Japan
}

\begin{abstract}
A viscous one-dimensional compressible pipe flow under gravity effect is studied analytically. The compressible one-dimensional pipe flow with friction is called Fanno flow and the solution is given by analytical formula. In gas dynamics, the gravity effect is minimal and it is not included in the equations. However, it was shown by the present author that the elevation of a pipe could change the flow conditions in a one-dimensional compressible potential flow under gravity. The sonic condition is reached at the maximum height for an inviscid pipe flow. In this paper, the gravity effect is extended to the viscous onedimensional pipe flow. Subsonic-supersonic transition is also possible by up and down of the pipe as in the inviscid flow, and it is found that the sonic condition deviates from the peak position of the pipe.
\end{abstract}

\section{Introduction}

In gas dynamics, the gravity effect is negligible and not included in the governing equations. However, the effect is evident in astrophysics, i.e. Bondi flow [1], [2]. It was also shown that an inviscid compressible onedimensional pipe flow could be accelerated from subsonic to supersonic by the elevation of the pipe in theory [3]. Although the gravity effect is not evident in an ordinary air flow, it can be noticeable near the sonic condition and in the low acoustic velocity like cryogenics. This sonic condition occurs at the peak location of a pipe and the gravity has a similar effect to that of the throat of a Laval nozzle [3].

In order to apply this gravity effect in the real world problem, the viscous effect should be taken into account. A one-dimensional pipe flow with friction is called Fanno flow and the analytical solution is available [4], [5]. In the present analysis, the gravity term is added to the Fanno flow equations.

\section{Governing equations}

\subsection{Governing equations}

The continuity equation for a steady one-dimensional compressible pipe flow in Fig. 1 is

$$
\rho u=\rho^{*} u^{*}
$$

where $u$ is the velocity, $\rho$ is the density and the symbol * denotes the sonic condition. The momentum equation is given by

$$
\rho u d u+d p+\frac{4 C_{f}}{D} \cdot \frac{1}{2} \rho u^{2} \cdot d l+\rho g \cdot d z=0
$$

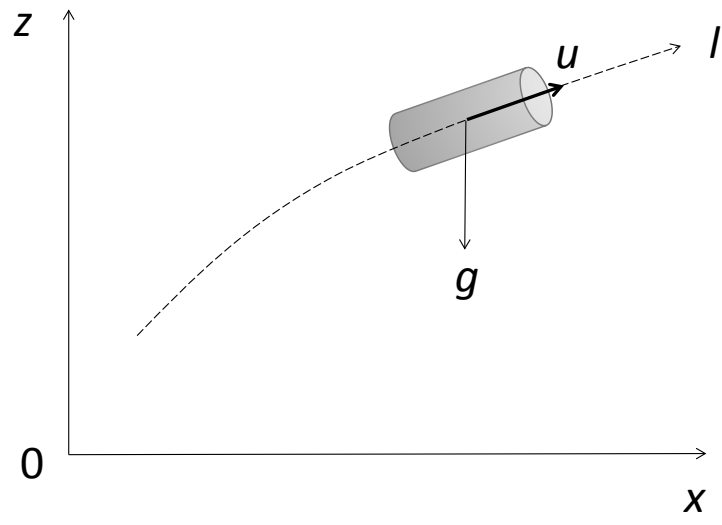

Fig. 1. Pipe coordinate system

where $C_{f}$ is the average skin friction coefficient, $D$ is the pipe diameter, $g$ is the gravitational acceleration, $l$ is the coordinate along the pipe, $p$ is the static pressure, $x$ is the horizontal coordinate and $z$ is the vertical coordinate.

The enetgy equation becomes

$$
\frac{1}{2} u^{2}+\frac{a^{2}}{\gamma-1}+g z=\frac{1}{2} \frac{\gamma+1}{\gamma-1} a^{* 2}+g z^{*}
$$

where $a$ is the local accoustic velocity. Equation (3) is the Bernoulli's equation and the right hand side of Eq.(3) is constant.

The equation of state is given by

$$
p=\rho R T
$$

From the definition of local Mach number $M$

\footnotetext{
* Corresponding author: etsuo.morishita@meisei-u.ac.jp
} 


$$
M^{2}=\frac{u^{2}}{a^{2}}=\frac{u^{2}}{\gamma R T}
$$

where $R$ is the gas constant and $\gamma$ is the specific heat ratio.

\subsection{Modified equations}

Equations (1)-(5) are modified as follow:

$$
\begin{aligned}
& \frac{d \rho}{\rho}+\frac{d u}{u}=0 \\
& \gamma M^{2} \frac{d u}{u}+\frac{d p}{p}+\frac{4 C_{f}}{D} \cdot \frac{1}{2} \gamma M^{2} \cdot d l+\gamma \frac{g d z}{a^{2}}=0 \\
& (\gamma-1) M^{2} \frac{d u}{u}+\frac{d T}{T}+(\gamma-1) \frac{g d z}{a^{2}}=0 \\
& \frac{d p}{p}=\frac{d \rho}{\rho}+\frac{d T}{T}
\end{aligned}
$$$$
\frac{d M^{2}}{M^{2}}=2 \frac{d u}{u}-\frac{d T}{T}
$$

From Eqs. (7) and (8) with Eqs. (6), (9) and (10),

$$
\left(M^{2}-1\right) \frac{d u}{u}+\frac{4 C_{f}}{D} \cdot \frac{1}{2} \gamma M^{2} \cdot d l+\frac{g d z}{a^{2}}=0
$$

For an inviscid flow with $C_{f}=0$,

$$
\left(M^{2}-1\right) \frac{d u}{u}+\frac{g d z}{a^{2}}=0
$$

Equation (12) shows that $d z=0$ at the sonic condition $M=1$. This implies the sonic condition occurs at the pipe peak location $z=z^{*}$. For the viscous case at $M=1$,

$$
\frac{d z}{d l}=-\frac{4 C_{f}}{D} \cdot \frac{1}{2} \gamma M^{2} \cdot \frac{a^{2}}{g}
$$

and the sonic condition is reaced after the pipe peak elevation.

From Eqs.(6)-(10), the following relations are derived.

$$
\frac{d u}{u}=-\frac{d \rho}{\rho}=\frac{1}{2} \frac{1}{1+\frac{\gamma-1}{2} M^{2}} \frac{d M^{2}}{M^{2}}-\frac{\frac{\gamma-1}{2}}{1+\frac{\gamma-1}{2} M^{2}} \frac{g d z}{a^{2}}
$$$$
\frac{d p}{p}=-\frac{1}{2} \frac{1+(\gamma-1) M^{2}}{1+\frac{\gamma-1}{2} M^{2}} \frac{d M^{2}}{M^{2}}-\frac{\frac{\gamma-1}{2}}{1+\frac{\gamma-1}{2} M^{2}} \frac{g d z}{a^{2}}
$$

$$
\begin{aligned}
& \frac{d T}{T}=-\frac{\frac{\gamma-1}{2}}{1+\frac{\gamma-1}{2} M^{2}} \frac{d M^{2}}{M^{2}}-\frac{\gamma-1}{1+\frac{\gamma-1}{2} M^{2}} \frac{g d z}{a^{2}} \\
& \frac{d s}{R}=\frac{\gamma}{\gamma-1} \frac{d T}{T}-\frac{d p}{p}=-\frac{d p_{0}}{p_{0}}=\frac{1}{2} \gamma M^{2} \cdot \frac{4 C_{f}}{D} \cdot d l
\end{aligned}
$$

where $p_{0}$ is the local total pressure and $s$ is the specific entropy. The local total pressure $p_{0}$ can be obtained from the local static pressure $p$ by assuming isentropic compression. The first terms of the right hand side of Eqs.(14)-(16) correspond to the Fanno flow solutions [4], [5], respectively.

\subsection{Solutions in Mach numbers}

From Eqs. (8) and (10),

$$
\frac{d M^{2}}{M^{2}}=2\left(1+\frac{\gamma-1}{2} M^{2}\right) \frac{d u}{u}+(\gamma-1) \frac{g d z}{a^{2}}
$$

From Eqs.(11) and (18), the gravitational terms can be eliminated, and

$\frac{d u}{u}=-\frac{d \rho}{\rho}=\frac{4 C_{f}}{D} \cdot \frac{1}{2} \frac{\gamma-1}{\gamma+1} \gamma M^{2} \cdot d l+\frac{1}{\gamma+1} \frac{d M^{2}}{M^{2}}$

Similarly,

$\frac{d p}{p}=\frac{d u}{u}-\frac{d M^{2}}{M^{2}}=\frac{4 C_{f}}{D} \cdot \frac{1}{2} \frac{\gamma-1}{\gamma+1} \gamma M^{2} \cdot d l-\frac{\gamma}{\gamma+1} \frac{d M^{2}}{M^{2}}$

$\frac{d T}{T}=2 \frac{d u}{u}-\frac{d M^{2}}{M^{2}}=\frac{4 C_{f}}{D} \frac{\gamma-1}{\gamma+1} \gamma M^{2} \cdot d l-\frac{\gamma-1}{\gamma+1} \frac{d M^{2}}{M^{2}}$

From Eqs. (17), (19), (20), (21) and (3)

$$
\begin{aligned}
& \ln \frac{u}{u^{*}}=4 C_{f} \cdot \frac{1}{2} \frac{\gamma(\gamma-1)}{\gamma+1} \cdot \int_{l_{*} / D}^{l_{M} / D} M^{2} \cdot d\left(\frac{l}{D}\right)+\frac{1}{\gamma+1} \ln M^{2} \\
& \left(=\ln \frac{\rho^{*}}{\rho}\right)
\end{aligned}
$$

$\ln \frac{p}{p^{*}}=4 C_{f} \cdot \frac{1}{2} \frac{\gamma(\gamma-1)}{\gamma+1} \int_{l_{*} / D}^{l / D} M^{2} \cdot d\left(\frac{l}{D}\right)-\frac{\gamma}{\gamma+1} \ln M^{2}$

$\ln \frac{T}{T^{*}}=4 C_{f} \cdot \frac{\gamma(\gamma-1)}{\gamma+1} \cdot \int_{l^{*} / D}^{l / D} M^{2} \cdot d\left(\frac{l}{D}\right)-\frac{\gamma-1}{\gamma+1} \ln M^{2}$

$$
\begin{aligned}
& \frac{s-s^{*}}{R}=4 C_{f} \cdot \frac{\gamma}{2} \cdot \int_{l^{*} / D}^{l / D} M^{2} \cdot d\left(\frac{l}{D}\right) \\
& \frac{g\left(z-z^{*}\right)}{a^{* 2}}=\frac{1}{2} \frac{\gamma+1}{\gamma-1}-\frac{1}{2}\left(\frac{u}{u^{*}}\right)^{2}-\frac{1}{\gamma-1} \frac{T}{T^{*}}
\end{aligned}
$$


where $l^{*}$ is the pipe sonic point. Equations (22)-(26) do not necessarily give the flow solutions directly. However, the pipe vertical coordinate $z$ relative to $z^{*}$ can be calculated from Eq.(26) with Eqs.(22) and (24) when the Mach number distribution is assumed.

\section{Mach number distribution}

\subsection{Inviscid flow}

In inviscid flows, the skin friction coefficient $C_{f}=0$ and from Eqs. (22) - (26) [3],

$$
\begin{aligned}
& \frac{u}{u^{*}}=\frac{\rho^{*}}{\rho}=M^{\frac{2}{\gamma+1}} \\
& \frac{p}{p^{*}}=M^{\frac{2 \gamma}{\gamma+1}} \\
& \frac{T}{T^{*}}=\frac{1}{M^{2 \frac{\gamma-1}{\gamma+1}}} \\
& \frac{s-s^{*}}{R}=0 \\
& \frac{z-z^{*}}{\left(\frac{a^{* 2}}{g}\right)}=\frac{1}{2} \frac{\gamma+1}{\gamma-1}-\frac{1}{2} M^{\frac{4}{\gamma+1}}-\frac{1}{\gamma-1} \frac{1}{M^{2 \frac{\gamma-1}{\gamma+1}}}
\end{aligned}
$$

In this case, Mach number $M$ can be calculated for a given $\left(z-z^{*}\right) /\left(a^{* 2} / g\right)$ from Eq.(31).

Figue 2 shows the Mach number distribution for $\gamma=1.4$ and

$$
\frac{z-z^{*}}{\left(\frac{a^{* 2}}{g}\right)}=-1+\exp \left[-3\left(\frac{x}{D}\right)^{2}\right]
$$

The elevation of pipe position has the same effect as that of the cross section of a Lavel nozzle. The sonic condition occurs at the peak height of the pipe.

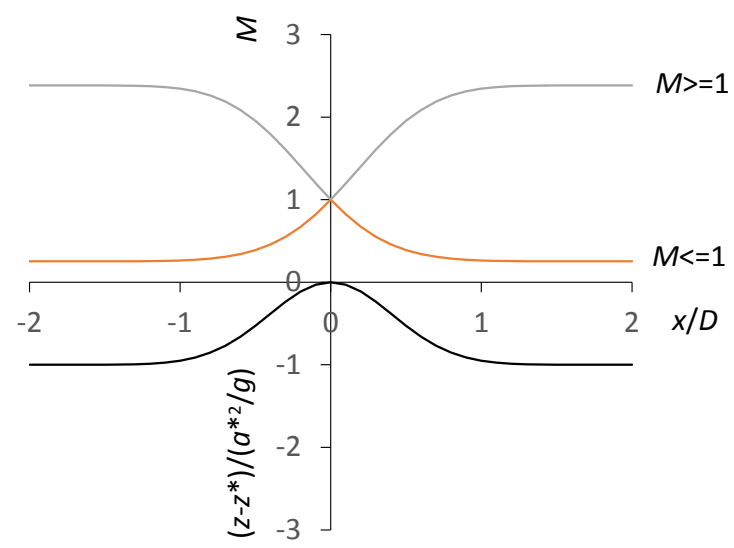

Fig. 2. Mach number distribution of inviscid pipe flow $\gamma=1.4$

\subsection{Viscous flow acceleration}

The viscous flow solutions Eqs. (22)-(26) are effective when the Mach number distribution is assumed. For example, a simple distribution from subsonic to supersonic transition can be given as follows:

$$
M^{2}=1+\frac{l}{D} \quad\left(\frac{l}{D}>-1\right)
$$

where

$$
\frac{l^{*}}{D}=0 \quad(M=1)
$$

The integral in Eqs. (22)-(25) becomes

$$
\int_{l^{\prime} / D}^{l / D} M^{2} \cdot d\left(\frac{l}{D}\right)=\left(\frac{l}{D}\right)+\frac{1}{2}\left(\frac{l}{D}\right)^{2}
$$

Then, Eqs. (22)-(26) become explicit function of $l / D$ and the pipe elevation Eq.(26) is obtained inversely.

Figure 3 shows the pipe geometry for the given Mach number distribution with skin friction coefficients as parameters.

As mentioned in Eq. (13) the subsonic-supersonic transition occurs at $l / D=l^{*} / D=0$ after the peak elevation of the pipe for $C_{f}>0$, while the inviscid flow reaches the sonic condition at the peak elevation of the pipe. It means that the flow has to be accelerated by gravity to overcome the pipe friction to be supersonic.

From Eq. (11), it is clear that the following equation is satisfied at the pipe peak position $d z=0$.

$$
\left(M^{2}-1\right) \frac{d u}{u}+\frac{4 C_{f}}{D} \cdot \frac{1}{2} \gamma M^{2} \cdot d l=0
$$

From Fig.3, the peak location of the pipe moves upstream as the skin friction coefficient $C_{f}$ increases.

Figure 4 shows the flow properties for $C_{f}=0.05$ in Fig.3. The temperature ratio $T / T^{*}$ rises gradually $l / D>0.9$ for $C_{f}=0.05$, while it decreases monotonically for the inviscid flow.

\subsection{Viscous flow deceleration}

Flow deceleration is also studied analytically. The Mach number might be assumed as follows:

$$
M^{2}=1-\frac{l}{D} \quad\left(\frac{l}{D}<1\right)
$$

The integral in Eqs. (22)-(25) becomes

$$
\int_{l^{\prime} / D}^{l / D} M^{2} \cdot d\left(\frac{l}{D}\right)=\left(\frac{l}{D}\right)-\frac{1}{2}\left(\frac{l}{D}\right)^{2}
$$




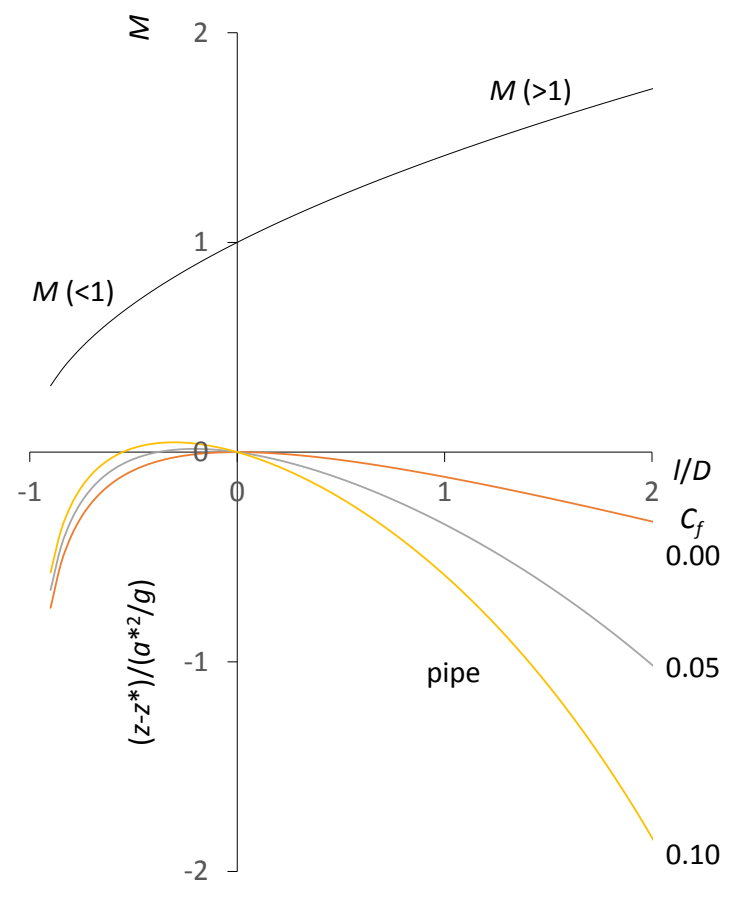

Fig. 3. Pipe geometry for $M=\sqrt{1+(l / D)}$ and $\gamma=1.4$

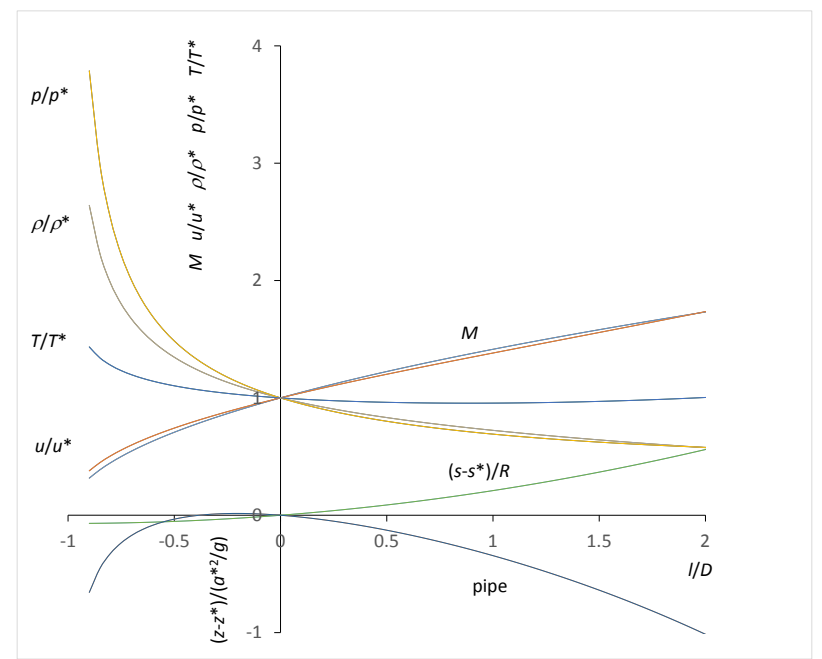

Fig. 4. Flow properties for $C_{f}=0.05$ in Fig. 2

Eqs. (22)-(26) become explicit function of $l / D$ and the pipe elevation Eq.(26) is obtained inversely as before.

Figure 5 shows the pipe geometry as a function of $l / D$ for the given Mach number distribution with the several different values of $C_{f}$. The deceleration of supersonic flow requires an ascent for small values of $C_{f}$, while a continuous descent is necessary to decelerate the flow for large values of $C_{f}$. This behaviour can be explained from Eq. (11). From Eq. (11)

$$
\frac{g d z}{a^{2}}=-\left(M^{2}-1\right) \frac{d u}{u}-\frac{4 C_{f}}{D} \cdot \frac{1}{2} \gamma M^{2} \cdot d l
$$

Equation (33) can be negative for large values of $C_{f}$ even for decelerating supersonic flow $(d u<0, M>1)$.

Flow properties are shown in Fig. 6 for $C_{f}=0.05$ in Fig 5.

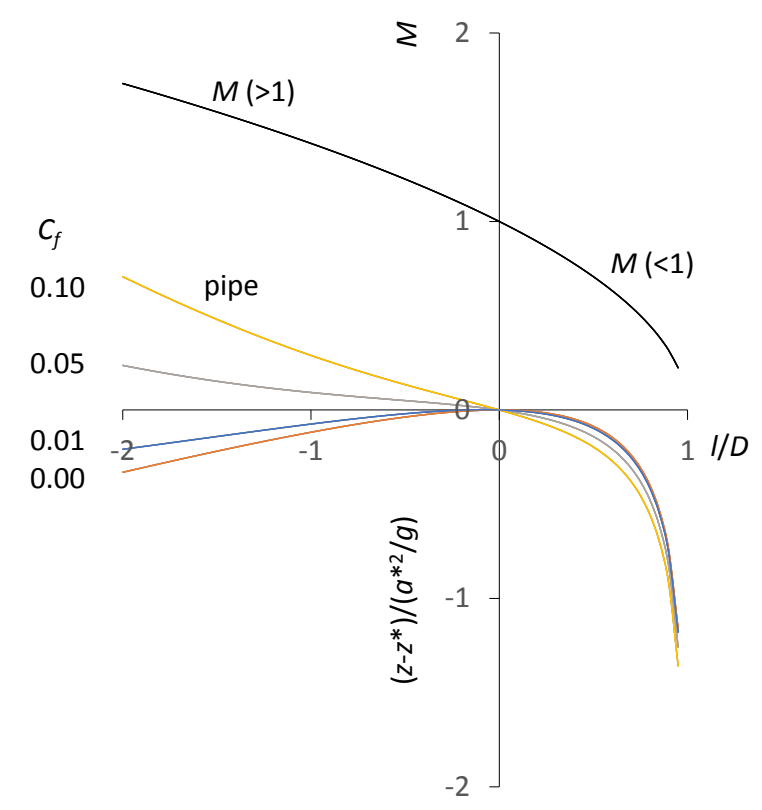

Fig. 5. Pipe geometry for $M=\sqrt{1-(l / D)}$ and $\gamma=1.4$

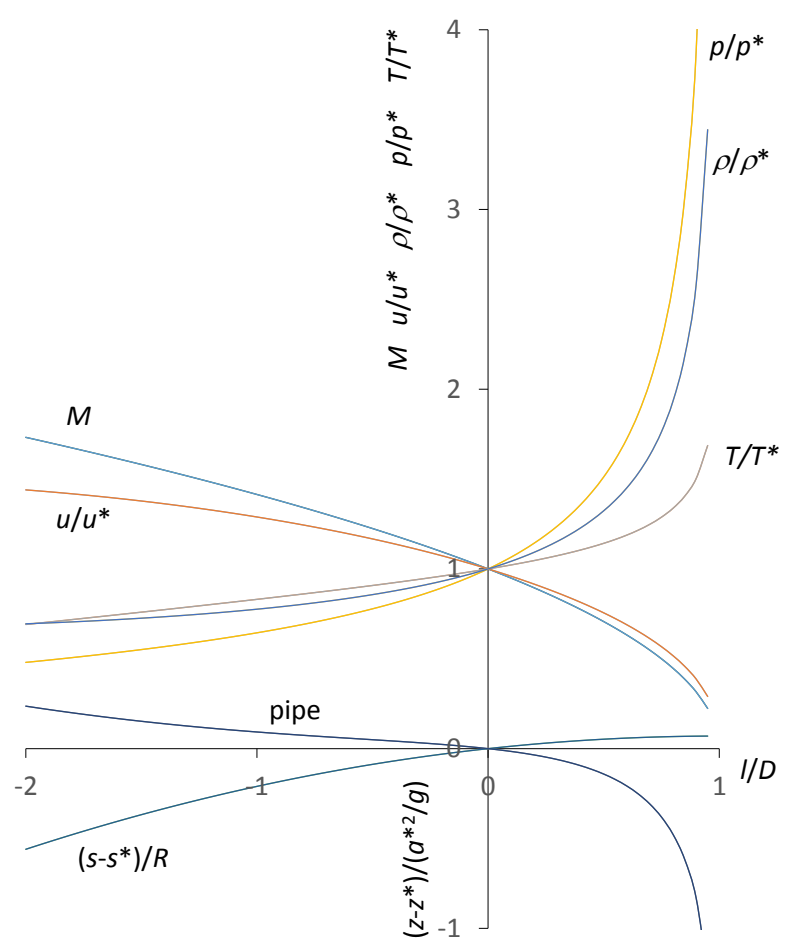

Fig. 6. Flow properties for $C_{f}=0.05$ in Fig. 3 


\section{Engineering application}

Subsonic-supersonic transition in a constant sectional area pipe by gravity effect is important from an engineering point of view.

Figure 7 shows an inviscid pipe flow with $g=9.8 \mathrm{~m} / \mathrm{s}^{2}$ and $a^{*}=100 \mathrm{~m} / \mathrm{s}$ derived from Fig. 2 . So the subsonic flow can be accelerated to a supersonic one with up and down of about $10 \mathrm{~m}$ around the peak of the pipe. The Mach number solely depends the height of the pipe $z-z^{*}$.

For $g=9.8 \mathrm{~m} / \mathrm{s}^{2}$ and $a^{*}=100 \mathrm{~m} / \mathrm{s}$, a subsonic flow of $M \approx 0.25$ can be acelerated to a supersonic flow of $M \approx 2.4$ by $z-z^{*} \approx 1000 \mathrm{~m}$ in Fig. 2 . The scale of the height is that of an planetary flow [6].

As for the viscous pipe flow solutions, the Mach number distribution is assumed along the pipe length $l$. From Fig.1, geometric relations is

$$
\Delta l^{2}=\Delta x^{2}+\Delta z^{2}
$$

and therefore the following conditions must be satisfied to obtain the physically proper solution.

$$
-1 \leq \frac{d z}{d l}=\frac{\left(\frac{a^{* 2}}{g}\right)}{D} \frac{d\left[\frac{\left(z-z^{*}\right)}{\left(\frac{a^{* 2}}{g}\right)}\right]}{d\left(\frac{l}{D}\right)} \leq 1
$$

Figure 8 shows an example of the viscous pipe flow with gravity derived form Fig.3, where the pipe diamater is given as $D=500 \mathrm{~m}$ to fit the geometric condition $|d z / d l| \leq 1$ with $g=9.8 \mathrm{~m} / \mathrm{s}^{2}, a^{*}=100 \mathrm{~m} / \mathrm{s} \quad, \quad$ and $C_{f}=0.05$, althogh $D$ is not a realistic value as an engineering problem. However, it might be possible for a planetary flow [6].

Note that lateral coodinate is tranformed to $x$ from $l$ measured along the pipe in Fig.8.

\section{Concluding remarks}

Compressible pipe flow with friction and gravity is modelled analytically. The viscous pipe flow is known as Fanno flow and choke occurs at the exit of the pipe. To accelerate a flow from subsonic to supersonic by gravity is theoretically possible for an inviscid pipe flow as previously shown by the present author. The subsonic-supersonic transition is also possible for the viscous pipe flow with gravity. The sonic condition is reached after the pipe peak elevation in this case. For deceleration from a supersonic flow to a subsonic one, there is no peak for larger pipe frictions, while an

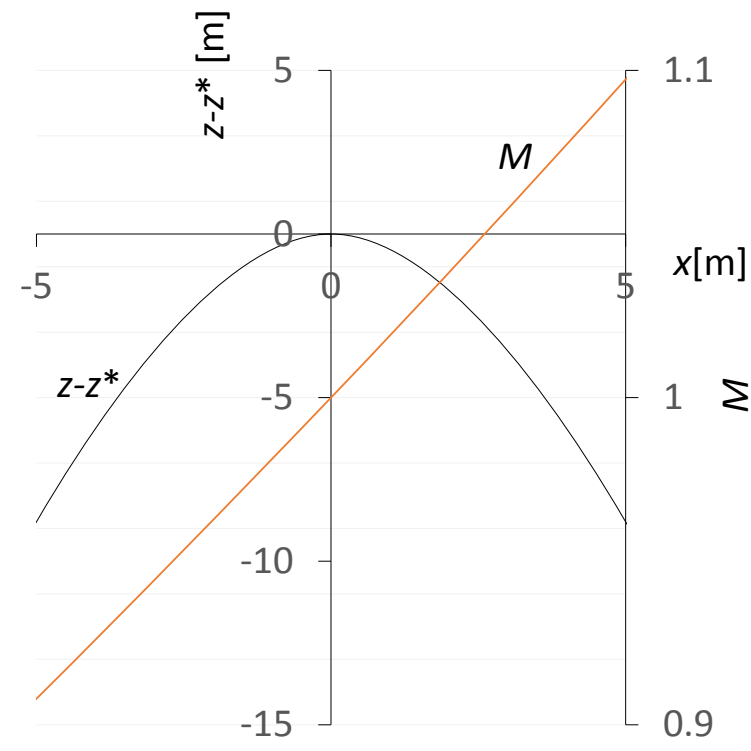

Fig. 7. Inviscid pipe flow subsonic-supersonic transition by gravity $g=9.8 \mathrm{~m} / \mathrm{s}^{2}$ and $a^{*}=100 \mathrm{~m} / \mathrm{s}$

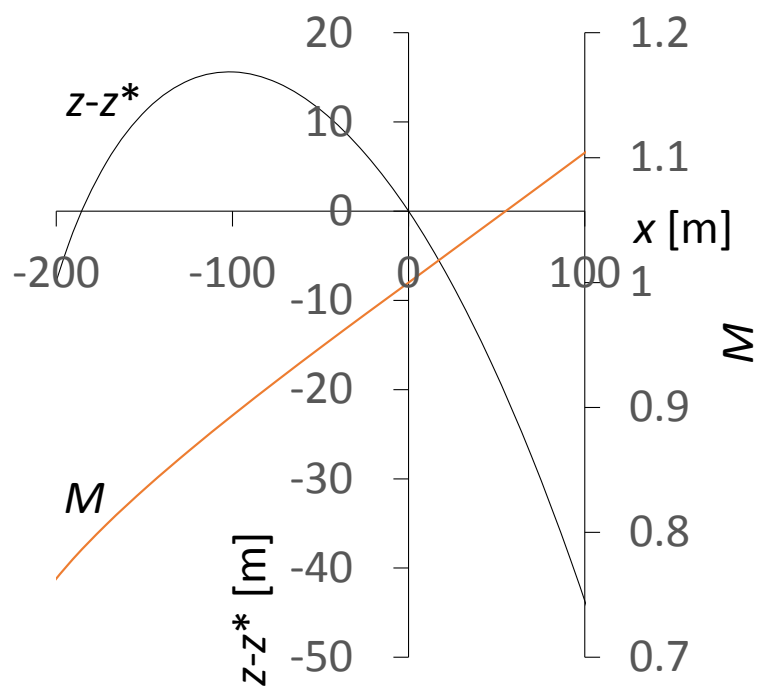

Fig. 8. Viscous pipe flow subsonic-supersonic transition by gravity for $g=9.8 \mathrm{~m} / \mathrm{s}^{2}, a^{*}=100 \mathrm{~m} / \mathrm{s}, C_{f}=0.05$ and $D=500 \mathrm{~m}$ in Fig. 3

inviscid supersonic flow goes upwards to decelerate to the sonic condition and downwards for subsonic deceleration.

The subsonic-supersonic transition might be possible by up and down of a pipe for both inviscid and viscous flows in theory. Although it is not an easy task to achieve this condition in a laboratory scale, it could be possible in cryogenics, planetary and astrophysics conditions. 


\section{References}

1. H. Bondi, H., Mon. Not. R. Astron. Soc. 112, 95 (1952)

2. S. N. Shore, S.N, An Introduction to Astrophysical Hydrodynamics (Academic Press, San Diego, 1992)

3. E. Morishita, Proc. WEC Vol III, London (2013)

http:// www.iaeng.org/ publication/ WCE 2013/WCE2013_pp1739-1742.pdf

4. M. A. Saad, Compressible Fluid Flow (Prentice-Hall, Englewood Cliffs, 1993)

5. J. D. Anderson, Modern Compressible Flow: With Historical Perspective (McGraw-Hill, New York, 2004)

6. https:// svs.gsfc.nasa.gov/11349D 